\title{
Erradicación de Helicobacter pylori en pacientes con dispepsia funcional
}

\author{
Helicobacter pylori eradication in patients with functional dyspepsia
}

Josefina Chiodia

\begin{abstract}
Resumen
La dispepsia constituye un motivo de consulta frecuente en atención primaria. A propósito de un paciente con diagnóstico de dispepsia funcional, la autora se plantea si el tratamiento de erradicación del Helicobacter pylori podría mejorar los síntomas. Luego de una búsqueda rápida se encontró evidencia que señala que el tratamiento de la infección por este germen podría ser beneficiosa para aliviar los síntomas de la dispepsia funcional a largo plazo, aunque con mayor riesgo de efectos adversos, por lo que otros tratamientos alternativos continúan siendo ser una opción válida en el manejo de los pacientes con este problema de salud.
\end{abstract}

Palabras clave: Dispepsia, Infecciones por Helicobacter, Helicobacter pylori. Keywords: Dyspepsia, Helicobacter Infections, Helicobacter pylori.

Chiodi J. Erradicación de Helicobacter pylori en pacientes con dispepsia funcional. Evid Actual Pract Ambul. 2020;23(3):e002070.

\section{Escenario clínico}

Concurre a la consulta un paciente varón de 61 años de edad, preocupado por la aparición reciente de síntomas gastrointestinales: pirosis, dolor postprandial en epigastrio. Se solicita una videoendoscopía digestiva alta (VEDA), que informa una mucosa gástrica congestiva sin lesiones ulcerosas, con Helicobacter pylori (Hp) positivo.

Se interpreta el cuadro como dispepsia funcional sin enfermedad ulceropéptica, y el médico tratante se plantea si el tratamiento para erradicación de Hp mejoraría los síntomas del paciente.

\section{Pregunta que generó el caso}

En pacientes adultos con dispepsia funcional y diagnóstico de infección por Helicobacter pylori (Población), ¿el tratamiento antibiótico para erradicación de esta bacteria (Intervención) vs. no realizar tratamiento de erradicación (Comparación), mejoraría los síntomas gastrointestinales (Outcome)?

\section{Algunos datos sobre la dispepsia y su relación con la infección por Helicobacter pylori}

La dispepsia se define como el dolor o malestar persistente o recurrente centrado en la parte superior del abdomen, y constituye un motivo de consulta muy frecuente al médico de familia. Las principales causas de dispepsia son los trastornos funcionales (50\% de los casos), la enfermedad por reflujo gastroesofágico $(30 \%)$ y la úlcera gastroduodenal $(20 \%)^{1}$. Para definir a la dispepsia como funcional se requiere la ausencia de una enfermedad que pueda explicar estos síntomas, incluyendo una VEDA normal ${ }^{2}$.

La infección por Hp representa la principal causa de úlceras gastroduodenales ${ }^{3}$. Estudios de prevalencia muestran que en los países en vías de desarrollo la prevalencia de Hp puede llegar a ser muy alta, superando en algunos casos el $70 \%$, y contrastando con la baja prevalencia de los países desarrollados. En Argentina, los estudios documentaron tasas que oscilan entre $52 \%$ y $56 \%$ en adultos sanos ${ }^{4}$. Asimismo, la infección varía entre grupos poblacionales, asociándose con un bajo nivel socioeconómico y educativo, así como con la falta de acceso al agua potable. Sin embargo, si tenemos en cuenta que sólo 15 a $20 \%$ de los pacientes infectados desarrollarán una úlcera, el índice de complicaciones es bajo. Otras complicaciones menos frecuentes relacionadas con la infección por $\mathrm{Hp}$ incluyen el adenocarcinoma y el linfoma gástricos ${ }^{3}$.
No caben dudas acerca de la indicación de tratamiento para erradicación del $\mathrm{Hp}$ en pacientes con dispepsia ulcerosa, ya que se ha demostrado una franca reducción en la recurrencia de esta entidad. Otras indicaciones claras para este tratamiento son el antecedente de úlcera, el linfoma gástrico MALT de bajo grado o la resección por cáncer gástrico temprano ${ }^{1}$. Sin embargo, no están tan claros los beneficios que puede aportar la erradicación en pacientes con dispepsia funcional. Una revisión sistemática Cochrane publicada en 2006 había documentado una reducción del riesgo relativo de 10\% (Intervalo de Confianza [IC] del $95 \%$ 6 a $14 \%$ ) en el grupo de terapia de erradicación del Hp, comparado con placebo, con un Número Necesario a Tratar (NNT) estimado de 14 pacientes. Sin embargo, en 12 de 17 ensayos clínicos incluidos en esa revisión no se había encontrado un beneficio significativo, lo que podría explicarse por el bajo número de pacientes incluidos, y concluía que era necesario contar con más investigación para determinar el balance de beneficios y riesgos de esta intervención ${ }^{5}$.

\section{Estrategia de búsqueda}

Se realizaron búsquedas en Medline utilizando combinaciones de términos Mesh (Dyspepsia/drug therapy, Dyspepsia/therapy, Helicobacter pylori) y lenguaje no controlado (functional, dys pepsia, helicobacter). Se aplicaron los filtros 'adult' y 'humans' y se limitó la búsqueda a los últimos 10 años. Adicionalmente, se revisaron las listas de artículos relacionados de los registros relevantes.

\section{Resumen de la evidencia}

Se seleccionaron dos revisiones sistemáticas ${ }^{6,7}$ que parecían responder a la pregunta que motivó este trabajo.

\section{Du LJ, et al. Helicobacter pylori eradication therapy for functional dyspepsia: Systematic review and meta-analysis. World J Gastroenterol. 2016;22(12):3486-95}

Objetivo: Esta revisión sistemática ${ }^{6}$ se propuso evaluar los beneficios de la erradicación del $\mathrm{Hp}$ en pacientes con dispepsia funcional.

Estrategia de búsqueda: se realizó en EMBASE, PubMed y la biblioteca Cochrane hasta 2015. Se incluyeron ensayos clínicos aleatorizados (ECA) que evaluaron la eficacia y seguridad de terapias de erradicación de $\mathrm{Hp}$ en pacientes con dispepsia funcional, publicados en inglés.

Resultados: se incluyeron un total de $25 \mathrm{ECA}$, con un total de 5.555 pacientes con dispepsia funcional y diagnóstico de $\mathrm{Hp}$ posi-

\footnotetext{
a Servicio de Medicina Familiar y Comunitaria, Hospital Italiano de Buenos Aires. josefina.chiodi@hospitalitaliano.org.ar
} 
tivo (prueba de urea en el aire espirado, histología o prueba rápido de ureasa). Veintitrés estudios evaluaron los beneficios de la terapia de erradicación para la mejoría sintomática. La erradicación de Hp representó una mejoría sintomática en el seguimiento mayor a un año (Riesgo Relativo [RR] 1,24; Intervalo de Confianza [IC] del $95 \% 1,12$ a 1,37), pero no hubo mejoría en el seguimiento a corto plazo (menos de un año) (RR 1,26; IC 95\% 0,83 a 1,92). Siete estudios encontraron beneficios en cuanto a calidad de vida (Diferencia de Medias Estandarizada [DME] de -0,01 IC $95 \%-0,11$ a 0,08), mientras que ocho estudios demostraron que el tratamiento para erradicación aumenta la chance de necesidad de tratamiento de efectos adversos (RR 2,02; IC $95 \%$ 1,12 a 3,65). Seis estudios observaron que esta terapia reduce el desarrollo de úlcera péptica en comparación con no realizar tratamiento (RR 0,35; IC $95 \%$ 0,18 a 0,68). Diez estudios mostraron que los pacientes que recibieron esta terapia tuvieron mayores probabilidades de resolución histológica de la gastritis crónica en comparación con quienes no la recibieron (RR 7,13; IC 95\% 3,68 a 13,81).

Conclusiones de los autores: la erradicación del Hp tendría un ligero beneficio en la reducción de síntomas, y también disminuiría el riesgo de desarrollar enfermedad ulceropéptica. Sin embargo, se debe tener en cuenta la mayor incidencia de efectos adversos relacionados con el tratamiento, y que no se han demostrado beneficios en la calidad de vida. Se pueden considerar otras terapias (antiácidos, procinéticos, antidepresivos) para el tratamiento de la dispepsia funcional.

\section{Kang SJ, et al. Helicobacter pylori eradication therapy for functional dyspepsia: a meta-analysis by region and $\mathrm{H}$. pylori prevalence.J Clin Med.2019;8,1324}

Objetivo: realizar un metanálisis ${ }^{7}$ que incluya ensayos aleatorios recientes y análisis de subgrupos sobre el efecto de la erradicación de $\mathrm{Hp}$ en el funcionamiento de la dispepsia realizados de acuerdo con la capacidad de respuesta completa, región geográfica y la prevalencia de $\mathrm{Hp}$.

Estrategia de búsqueda: se buscó en PubMed, EMBASE y la Biblioteca Cochrane hasta diciembre de 2018. Se incluyeron ECA que evaluaron el efecto de la erradicación de $\mathrm{Hp}$ en la dispepsia funcional. Se realizaron análisis de subgrupos por la medida de resultado, región y prevalencia de $\mathrm{Hp}$. Todos los datos fueron analizados con Review Manager 5.3.

Resultados: Se incluyeron 18 ECA. El grupo de erradicación de Hp mostró una mejora significativa de los síntomas en comparación con el grupo control (RR 1,18; IC $95 \% 1,07$ a 1,30). Hubo heterogeneidad moderada entre los estudios $\left(\mathrm{I}^{2}=34 \%\right)$ y el NNT fue 15 (IC $95 \%$ 10,5 a 25). La erradicación de Hp mejoró los síntomas dispépticos tanto en regiones de prevalencia baja (<50\%) como alta ( $\geq 50 \%)$, con RR 1,21 (IC $95 \% 1,02$ a 1,$44 ; I^{2}=49 \%$ ) y 1,17 (IC $95 \% 1,06$ a 1,29; $\left.\left.\right|^{2}=5 \%\right)$, respectivamente. En el análisis de estudios de Asia, sin embargo, el efecto no fue significativo (RR 1,14; IC $95 \%$ 0,99 a 1,33, $I^{2}=37 \%$ ).

Conclusión: la erradicación de Hp proporciona una significativa mejora de los síntomas en pacientes con dispepsia funcional independientemente de la prevalencia de $\mathrm{Hp}$. Sin embargo, en el análisis de estudios de Asia, la erradicación no mejoró significativamente los síntomas. En esta región, la erradicación de la dispepsia se puede individualizar.

\section{Conclusiones y recomendaciones}

Teniendo en cuenta estos resultados e intentando responder la pregunta disparadora, podríamos decir que aquellos pacientes que tienen $\mathrm{Hp}$ positivo presentan un ligero beneficio en cuanto a mejoría de sus síntomas al ser tratados con antibióticos para la erradicación de esta bacteria, con un NNT de 14 pacientes según la revisión Cochrane ${ }^{5}$ y de 15 en el meta-análisis más actualizado ${ }^{7}$ Estos resultados van en consonancia con lo recomendado por las guías norteamericanas, que proponen que aquellos pacientes con dispepsia que no tengan indicación de VEDA (es decir, menores de 60 años y sin banderas rojas), sean testeados para $\mathrm{Hp}$ con métodos no invasivos, y que aquellos que sean positivos reciban tratamiento para erradicación. Es decir, recomienda buscar activamente la infección por $\mathrm{Hp}^{8}$.

Algo similar ocurre con las guías europeas, que también recomiendan la estrategia 'evaluar y tratar', buscar y tratar aquellos pacientes colonizados, exceptuando a poblaciones con prevalencia de $\mathrm{Hp}$ menor al $10 \%$, donde las probabilidades de que la dispepsia pueda deberse a esta infección son muy bajas ${ }^{9}$

Algunas consideraciones que siempre surgen respecto a la aplicabilidad de estos resultados en nuestro medio, tienen relación con la prevalencia de infección por $\mathrm{Hp}$, sin embargo en el meta-análisis resumido de Kang et $a^{7}$ se mostró su eficacia en regiones con prevalencias mayores y menores al $50 \%$.

La estrategia de 'evaluar y tratar' en nuestro medio tiene otras cuestiones a resolver como: disponibilidad de las pruebas no invasivas recomendadas (test de urea espirada o antígeno en materia fecal), costo económico, efectos adversos, modificación de la microbiota intestinal, desarrollo de mayor resistencia bacteriana y posibilidad de reinfección.

Si bien la opción de 'evaluar y tratar' a estos pacientes es recomendable, se necesitarán más estudios para poder evaluar el impacto del tratamiento y erradicación del $\mathrm{Hp}$ en nuestro medio, los posibles beneficios de esta estrategia y la costoefectividad de la misma.

\section{Referencias}

1. Servicio de Medicina Familiar y Comunitaria del Hospital Italiano de Buenos Aires. Medicina Familiar y Práctica Ambulatoria. 3rd ed. Kopitowski K, Carrete P, Barani M, et al., editors. Buenos Aires: Editorial Médica Panamericana; 2016.

2. Stanghellini V, Chan FKL, Hasler WL, et al. Gastroduodenal Disorders. Gastroenterology. 2016;150(6):1380-1392. Available from: 10.1053/j.gastro. 2016.02.011.

3. Hospital Italiano de Buenos Aires Servicio de Medicina Familiar y Comunitaria . Dispepsia. In: Material Educativo PROFAM, Programa de educación continua en medicina familiar, ambulatoria y comunitaria. Buenos Aires, Argentina; 2020.

4. Corti RE. Helicobacter pylori: algunos aspectos epidemiológicos en Latinoamérica luego de un cuarto de siglo [Helicobacter pylori: some epydemiological questions in Latin America after twenty-five years]. Acta Gastroenterol Latinoam. 2009;39(3):175-176.

5. Moayyedi P, Soo S, Deeks J, et al. Eradication of helicobacter pylori for non-ulcer dyspepsia. Cochrane Database Syst Rev. 2000 ;(2):2096-2096. Available from: 10.1002/14651858.CD002096.

6. Du LJ, Chen BR, Kim JJ, et al. Helicobacter pylorieradication therapy for functional dyspepsia: Systematic review and meta-analysis. World J Gastroenterol. 2016;22(12):3486-3495. Available from: 10.3748/wjg.v22.i12.3486

7. Kang SJ, Park B, Shin CM. Helicobacter pylori Eradication Therapy for Functional Dyspepsia: A Meta-Analysis by Region and H. pylori Prevalence. J Clin Med. 2019;8(9):1324. Available from: doi.org/10.3390/jcm8091324.

8. Moayyedi PM, Lacy BE, Andrews CN, et al. ACG and CAG Clinical Guideline: Management of Dyspepsia. Am J Gastroenterol. 2017;112(7):9881013. Available from: 10.1038/ajg.2017.154.

9. Malfertheiner P, Megraud F, Morain CAO, et al. Management of Helicobacter pylori infection-the Maastricht V/Florence Consensus Report. Gut. 2017;66(1):6-30. Available from: 10.1136/gutjnl-2016-312288. 